\title{
Interpolation for Predefined Types
}

\author{
R $\breve{A} Z$ V AN DIACONESCU \\ Simion Stoilow Institute of Mathematics of the Romanian Academy \\ Received August 2008
}

\begin{abstract}
We give a logic-independent semantics for predefined (data) types within the categorical abstract model theoretic framework of the theory of institutions. For this semantics we develop a generic interpolation result which can be easily applied to various concrete situations from the theory and practice of specification and programming. Our study of interpolation is motivated by a number of important applications to computing science especially in the area of structured specifications.
\end{abstract}

\section{Introduction}

The practice of programming and specification relies quite a lot upon the use of predefined (data) types together with that of user defined types. The most common examples of predefined types include numbers (natural, integers, reals, etc.), Booleans, but also lists and arrays. These are usually built into the respective programming or specification systems. Another usage of predefined types may occur when using libraries, often implemented in a lower level language. A model theoretic semantics for predefined types was first discussed in (Goguen and Meseguer 1987) within the context of the (many sorted) equational logic programming paradigm. An important feature of that semantics was that it promised a smooth and elegant integration of constraint solving into ordinary (equational) logic programming. The semantics for predefined types discussed in (Goguen and Meseguer 1987) was fully developed at an abstract category-based level in (Diaconescu 1994; Diaconescu 2000). By that abstract approach to logic programming, at least at the denotational level, large parts of the constraint programming paradigm were realized as special cases of ordinary logic programming via Herbrand theorems for constraints logic programming obtained as instances of general abstract Herbrand theorems to the semantic framework for predefined types. All these have been further generalized to a fully logic-independent framework in (Diaconescu 2004b; Diaconescu 2008).

Here we update this semantics, in the sense of a mathematical simplification, but without reducing the level of generality. This is done at the same logic-independent level of previous works such as (Diaconescu 2000), given by the so-called institution theory (Goguen and Burstall 1992), which is a categorical abstract model theory that arose within specification theory as a response to the explosion in the population of logics in use there. The main concept of institution theory is that of 'institution', which constitutes a formal mathematical model theoretic definition for the informal concept of logical system; this definition includes syntax (signatures and sentences), semantics (models), and the satisfaction relation between them. Much of modern formal specification theory (see (Goguen and Burstall 1992; Sannella and Tarlecki 1988; Diaconescu et al. 
1993) among many other related references) and large parts of model theory (Diaconescu 2008), respectively, have been developed at the generic level of abstract institutions free of any commitement to the details of particular logics; these are called 'institution-independent' computing science and model theory, respectively. We thus introduce a generic way to define a (model theoretic) logic of predefined types on top of any given logical system which is captured as an institution. We show that the resulting logic turns out to be an institution too.

The emphasis of the current work is on the study of the interpolation properties of the logics (institutions) of predefined types. The main reason for this is that interpolation, besides being a notoriously important logical property, it also has a number of important applications in computing science especially in formal specification theory (Bergstra et al. 1990; Diaconescu et al. 1993; Dimitrakos 1998; Bicarregui et al. 2001; Veloso 1996; Borzyszkowski 2000) but also in data bases (ontologies) (Kutz and Mossakowski 2007), automated reasoning (Nelson and Oppen 1979; Oppen 1980), type checking (Jhala et al. 2007), model checking (Mcmillan 2005), and structured theorem proving (Amir and MCllraith 2000; McIlarith and Amir 2001). The main result of this paper is a general theorem lifting interpolation properties from the base institution to its corresponding institution of predefined types. The concept of interpolation for predefined types gets a straightforward definition due to the general concept of interpolation at the level of abstract institutions. This is one of the examples of the great benefits of developing concepts abstractly at an institution-independent level. The generic interpolation result for predefined types is illustrated with a couple of concrete examples involving logics common to specification and logic programming. Moreover, due to its generality, this can be applied to many other concrete situations. The proof of our generic interpolation theorem for predefined types requires a number of new general institution theoretic results together with a model amalgamation property for the institutions of predefined types, all these are also developed here.

The paper has the following structure:

1 A section on preliminaries introducing the institution-theoretic concepts needed by our work and developing some new general technical results used for proving the main result of this paper.

2 A section devoted to the construction of institutions of predefined types and the study of their model amalgamation properties.

3 The section in which we solve the interpolation problem for predefined types.

\section{Preliminaries}

In this section we recall briefly common technical concepts and results of institution-independent model theory needed by our current paper and we develop several new general technical results which we will use specifically for this work. The section contains three parts:

1 A succint recall of some basic category-theoretic notations and concepts used by our paper.

2 A succint presentation of the common institution-theoretic concepts and results needed by our paper. In this part we also develop a technical result which we will use specifically in the current paper.

3 The last part of this section is devoted to the generic concept of interpolation in the institutionindependent setting. It also contains a couple of technical results used specifically by our current work. 


\subsection{Categories}

We assume the reader is familiar with basic notions and standard notations from category theory; e.g., see (Mac Lane 1998) for an introduction to this subject. Here we recall very briefly some of them. By way of notation, $|\mathbb{C}|$ denotes the class of objects of a category $\mathbb{C}, \mathbb{C}(A, B)$ the set of arrows with domain $A$ and codomain $B$, and composition is denoted by ";" and in diagrammatic order. The category of sets (as objects) and functions (as arrows) is denoted by $\mathbb{S} e t$, and $\mathbb{C} a t$ is the category of all categories. ${ }^{\dagger}$ The opposite of a category $\mathbb{C}$ (obtained by reversing the arrows of $\mathbb{C}$ ) is denoted $\mathbb{C}^{\text {op }}$.

2.1.1. Comma categories. For any object $A$ in a category $|\mathbb{C}|$, the comma category $A / \mathbb{C}$ has arrows $f: A \rightarrow B$ as objects and $h \in \mathbb{C}\left(B, B^{\prime}\right)$ with $f ; h=f^{\prime}$ as arrows $f \rightarrow f^{\prime}$.

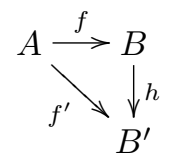

2.1.2. Grothendieck categories. An indexed category is a functor $B: I^{\mathrm{op}} \rightarrow \mathbb{C}$ at. Given an indexed category $B: I^{\text {op }} \rightarrow \mathbb{C} a t$, let $B^{\sharp}$ be the Grothendieck category having $\langle i, \Sigma\rangle$, with $i \in|I|$ and $\Sigma \in|B(i)|$, as objects and $\langle u, \varphi\rangle:\langle i, \Sigma\rangle \rightarrow\left\langle i^{\prime}, \Sigma^{\prime}\right\rangle$, with $u \in I\left(i, i^{\prime}\right)$ and $\varphi: \Sigma \rightarrow B(u)\left(\Sigma^{\prime}\right)$, as arrows. The composition of arrows in $B^{\sharp}$ is defined by

$\langle u, \varphi\rangle ;\left\langle u^{\prime}, \varphi^{\prime}\right\rangle=\left\langle u ; u^{\prime}, \varphi ; B(u)\left(\varphi^{\prime}\right)\right\rangle$.

\subsection{Institution-theoretic preliminaries}

Here we review briefly a series of institution-theoretic concepts and results that we are going to use for our work. These includes the definition of the concept of institution together with some examples, the concept of conservative signature morphism, and the institution-theoretic concepts of presentations, model amalgamation, liberality, as well as the institution-independent method of diagrams.

2.2.1. Basic notions Institutions have been defined by Goguen and Burstall in (Burstall and Goguen 1980), the seminal paper (Goguen and Burstall 1992) being printed after a delay of many years. Below we recall the concept of institution which formalises the intuitive notion of logical system, including syntax, semantics, and the satisfaction between them.

Definition 2.1 (Institutions). An institution $\mathcal{I}=\left(\operatorname{Sig}^{\mathcal{I}}, \operatorname{Sen}^{\mathcal{I}}, \operatorname{Mod}^{\mathcal{I}}, \models^{\mathcal{I}}\right)$ consists of

1 a category $\mathbb{S i g}^{\mathcal{I}}$, whose objects are called signatures,

2 a functor $\operatorname{Sen}^{\mathcal{I}}: \mathbb{S i g}^{\mathcal{I}} \rightarrow \mathbb{S e t}$, giving for each signature a set whose elements are called sentences over that signature,

3 a functor $\operatorname{Mod}^{\mathcal{I}}:\left(\mathbb{S i g}{ }^{\mathcal{I}}\right)^{\text {op }} \rightarrow \mathbb{C} \mathbb{A}$ giving for each signature $\Sigma$ a category whose objects are called $\Sigma$-models, and whose arrows are called $\Sigma$-(model $)$ morphisms, and

\footnotetext{
$\dagger$ Strictly speaking, this is only a 'quasi-category' living in a higher set-theoretic universe.
} 
4 a relation $\models_{\Sigma}^{\mathcal{I}} \subseteq\left|\operatorname{Mod}^{\mathcal{I}}(\Sigma)\right| \times \operatorname{Sen}^{\mathcal{I}}(\Sigma)$ for each $\Sigma \in\left|\operatorname{Sig}^{\mathcal{I}}\right|$, called $\Sigma$-satisfaction, such that for each morphism $\varphi: \Sigma \rightarrow \Sigma^{\prime}$ in $\mathbb{S} i g^{\mathcal{I}}$, the satisfaction condition

$$
M^{\prime} \models \Sigma_{\Sigma^{\prime}}^{\mathcal{I}} \operatorname{Sen}^{\mathcal{I}}(\varphi)(\rho) \text { iff } \operatorname{Mod}^{\mathcal{I}}(\varphi)\left(M^{\prime}\right) \models \frac{\mathcal{I}}{\Sigma} \rho
$$

holds for each $M^{\prime} \in\left|\operatorname{Mod}^{\mathcal{I}}\left(\Sigma^{\prime}\right)\right|$ and $\rho \in \operatorname{Sen}^{\mathcal{I}}(\Sigma)$.

We may denote the reduct functor $\operatorname{Mod}^{\mathcal{I}}(\varphi)$ by $-\uparrow_{\varphi}$ and the sentence translation $\operatorname{Sen}^{\mathcal{I}}(\varphi)$ by $\varphi(-)$. When $M=\left.M^{\prime}\right|_{\varphi}$ we say that $M$ is a $\varphi$-reduct of $M^{\prime}$, and that $M^{\prime}$ is a $\varphi$-expansion of $M$. When there is no danger of ambiguity, we may skip the superscripts from the notations of the entities of the institution; for example $\mathbb{S i g}{ }^{\mathcal{I}}$ may be simply denoted $\mathbb{S i g}$. Also, when the signature is clear we may omit it as subscript of the satisfaction relation $=$.

General assumption: We assume that all our abstract institutions are such that satisfaction is invariant under model isomorphism, i.e. if $\Sigma$-models $M, M^{\prime}$ are isomorphic, denoted $M \cong M^{\prime}$, then $M \models_{\Sigma} \rho$ iff $M^{\prime} \models_{\Sigma} \rho$ for all $\Sigma$-sentences $\rho$. This very basic assumption holds commonly in all concrete institutions of interest, including those discussed in our current paper.

Notation 2.1. For any $\Sigma$-model $M$ and any set $E$ of $\Sigma$-sentences by $M \models_{\Sigma} E$ we denote that for each $\rho \in E, M \mid=_{\Sigma} \rho$.

For $E$ and $E^{\prime}$ sets of $\Sigma$-sentences in an arbitrary institution by $E \models_{\Sigma} E^{\prime}$ we denote that for all $\Sigma$-models $M$, if $M \models_{\Sigma} E$ then $M \models_{\Sigma} E^{\prime}$. By $E \| E^{\prime}$ we denote the situation that $E$ and $E^{\prime}$ are semantically equivalent, i.e. that $E \models E^{\prime}$ and $E^{\prime} \models E$.

There are myriads examples of institutions from logic or computing science (see (Diaconescu 2008) for some of these). Below we present a couple of them which we are going to use as concrete benchmarks for our general results.

Example 2.1 (FOL). Let $F O L$ be the institution of first order logic with equality in its many sorted form.

Its signatures are triples $(S, F, P)$ consisting of

- a set of sort symbols $S$,

- a family $F=\left\{F_{w \rightarrow s} \mid w \in S^{*}, s \in S\right\}$ of sets of function symbols indexed by arities (for the arguments) and sorts (for the results), and

- a family $P=\left\{P_{w} \mid w \in S^{*}\right\}$ of sets of relation (predicate) symbols indexed by arities.

Signature morphisms map the three components in a compatible way. This means that a signature morphism $\varphi:(S, F, P) \rightarrow\left(S^{\prime}, F^{\prime}, P^{\prime}\right)$ consists of

- a function $\varphi^{\text {st }}: S \rightarrow S^{\prime}$,

- a family of functions $\varphi^{\mathrm{op}}=\left\{\varphi_{w \rightarrow s}^{\mathrm{op}}: F_{w \rightarrow s} \rightarrow F_{\varphi^{\mathrm{st}}(w) \rightarrow \varphi^{\mathrm{st}}(s)}^{\prime} \mid w \in S^{*}, s \in S\right\}$, and

- a family of functions $\varphi^{\mathrm{rl}}=\left\{\varphi_{w \rightarrow s}^{\mathrm{rl}}: P_{w} \rightarrow P_{\varphi^{\mathrm{st}}(w)}^{\prime} \mid w \in S^{*}, s \in S\right\}$.

Models $M$ for a signature $(S, F, P)$ are first order structures interpreting each sort symbol $s$ as a set $M_{s}$, each function symbol $\sigma$ as a function $M_{\sigma}$ from the product of the interpretations of the argument sorts to the interpretation of the result sort, and each relation symbol $\pi$ as a subset $M_{\pi}$ of the product of the interpretations of the argument sorts. By $|M|$ we denote $\left\{M_{s} \mid s \in S\right\}$ and we call it the universe of $M$ or the carrier set( $s$ ) of $M$. In order to avoid the existence of empty interpretations of the sorts, which may complicate unnecessarily our presentation, we assume 
that each signature has at least one constant (i.e. function symbol with empty arity) for each sort. A model homomorphism $h: M \rightarrow M^{\prime}$ is an indexed family of functions $\left\{h_{s}: M_{s} \rightarrow M_{s}^{\prime}\right\}_{s \in S}$ such that

- $h$ is an $(S, F)$-algebra homomorphism $M \rightarrow M^{\prime}$, i.e., $h_{s}\left(M_{\sigma}(m)\right)=M_{\sigma}^{\prime}\left(h_{w}(m)\right)$ for each $\sigma \in F_{w \rightarrow s}$ and each $m \in M_{w}$, and

- $h_{w}(m) \in M_{\pi}^{\prime}$ if $m \in M_{\pi}$ (i.e. $h_{w}\left(M_{\pi}\right) \subseteq M_{\pi}^{\prime}$ ) for each relation $\pi \in P_{w}$ and each $m \in M_{w}$. where $h_{w}: M_{w} \rightarrow M_{w}^{\prime}$ is the canonical component-wise extension of $h$, i.e. $h_{w}\left(m_{1}, \ldots, m_{n}\right)=$ $\left(h_{s_{1}}\left(m_{1}\right), \ldots, h_{s_{n}}\left(m_{n}\right)\right)$ for $w=s_{1} \ldots s_{n}$ and for each $1 \leq i \leq n, m_{i} \in M_{s_{i}}$.

For each signature morphism $\varphi$, the reduct $M^{\prime} \uparrow_{\varphi}$ of a model $M^{\prime}$ is defined by $\left(M^{\prime} \uparrow_{\varphi}\right)_{x}=$ $M_{\varphi(x)}^{\prime}$ for each sort, function, or relation symbol $x$ from the domain signature of $\varphi$.

Sentences are the usual first order sentences built from equational and relational atoms by iterative application of Boolean connectives and quantifiers. Sentence translations along signature morphisms just rename the sorts, function, and relation symbols according to the respective signature morphisms. They can be formally defined by induction on the structure of the sentences. While the induction step is straightforward for the case of the Boolean connectives it needs a bit of attention for the case of the quantifiers. For any signature morphism $\varphi:(S, F, P) \rightarrow\left(S^{\prime}, F^{\prime}, P^{\prime}\right)$,

$$
\operatorname{Sen}^{F O L}(\varphi)((\forall X) \rho)=\left(\forall X^{\varphi}\right) \operatorname{Sen}^{F O L}\left(\varphi^{\prime}\right)(\rho)
$$

for each finite block $X$ of variables for $(S, F, P)$. The variables need to be disjoint from the constants of the signature, also we have to ensure that $\operatorname{Sen}{ }^{F O L}$ thus defined is functorial indeed and that there is no overloading of variables (which in certain situations would cause a failure of the Satisfaction Condition). These may be formally achieved by considering that a variable for $(S, F, P)$ is a triple of the form $(x, s,(S, F, P))$ where $x$ is the name of the variable and $s \in S$ is the sort of the variable and that two different variables in $X$ have different names. We often abbreviate variables $(x, s,(S, F, P))$ by their name $x$. Then we let $(S, F+X, P)$ be the extension of $(S, F, P)$ such that $(F+X)_{w \rightarrow s}=F_{w \rightarrow s}$ when $w$ is non-empty and $(F+X)_{\rightarrow s}=F_{\rightarrow s} \cup$ $\{(x, s,(S, F, P)) \mid(x, s,(S, F, P)) \in X\}$ and we let $\varphi^{\prime}:(S, F+X, P) \rightarrow\left(S^{\prime}, F^{\prime}+X^{\varphi}, P^{\prime}\right)$ be the canonical extension of $\varphi$ that maps each variable $(x, s,(S, F, P))$ to $\left(x, \varphi(s),\left(S^{\prime}, F^{\prime}, P^{\prime}\right)\right)$.

The satisfaction of sentences by models is the usual Tarskian satisfaction defined recursively on the structure of the sentences.

\section{Example 2.2. (Horn clause logic with equality).}

This is the institution underlying the pure form of the (equational) logic programming paradigm and we denlote it by $H C L$. It is precisely the logic underlying the work of the seminal paper (Goguen and Meseguer 1987). HCL has the same signatures and sentences as FOL but the so-called Horn clauses as sentences. The Horn clauses are just FOL sentences of the form $(\forall X) H \Rightarrow C$ where $H$ is a finite conjunction of atoms and $C$ is atom. The satisfaction relation of $H C L$ is just the restriction of the satisfaction relation in $F O L$.

The following general technical concept is used for proving the main result of the current paper.

Definition 2.2 (Conservative signature morphisms). A signature morphism $\varphi: \Sigma \rightarrow \Sigma^{\prime}$ is conservative when each $\Sigma$-model has a $\varphi$-expansion. 
For example (see (Diaconescu 2008)) a FOL signature morphism is conservative when all its components are injective.

One of the most useful properties of conservative signature morphisms is given by the following fact which is an easy consequence of the satisfaction condition.

Fact 2.1. For any conservative signature morphism $\varphi: \Sigma \rightarrow \Sigma^{\prime}$ and any sets of $\Sigma$-sentences $E$ and $\Gamma$

$$
E \models_{\Sigma} \Gamma \text { if and only if } \varphi(E) \models_{\Sigma^{\prime}} \varphi(\Gamma) .
$$

2.2.2. Presentations In formal specification theory, institution-theoretic presentations capture the concept of basic (unstructured) specifications. Moreover, the concept of presentation is a quite useful construction in several model theoretic developments (Diaconescu 2008).

Definition 2.3 (Presentations). In any institution, a presentation is a pair $(\Sigma, E)$ consisting of a signature $\Sigma$ and a set $E$ of $\Sigma$-sentences. A presentation morphism $\varphi:(\Sigma, E) \rightarrow\left(\Sigma^{\prime}, E^{\prime}\right)$ is a signature morphism $\varphi: \Sigma \rightarrow \Sigma^{\prime}$ such that $E^{\prime} \models \varphi(E)$.

Fact 2.2. Presentation morphisms are closed under the composition given by the composition of the signature morphisms.

This fact opens the door for the general construction given by the following definition.

Definition 2.4 (The institution of the presentations). Let $\mathcal{I}=(\operatorname{Sig}$, Sen, Mod, $\models)$ be any institution. The institution of the presentations of $\mathcal{I}$, denoted by $\mathcal{I}^{\text {pres }}=\left(\mathbb{S} i g^{\text {pres }}\right.$, Sen $^{\text {pres }}$, Mod $\left.^{\text {pres }}, \models^{\text {pres }}\right)$, is defined by

- $\mathbb{S}_{i} g^{\text {pres }}$ is the category $\mathbb{P r e s}$ of presentations of $\mathcal{I}$,

- $\operatorname{Sen}^{\text {pres }}(\Sigma, E)=\operatorname{Sen}(\Sigma)$,

- $\operatorname{Mod}^{\text {pres }}(\Sigma, E)$ is the full subcategory of $\operatorname{Mod}(\Sigma)$ of those models which satisfy $E$, and

- for each $(\Sigma, E)$-model $M$ and $\Sigma$-sentence $e, M \models_{(\Sigma, E)}^{\text {pres }} e$ if and only if $M \models_{\Sigma} e$.

Fact 2.3. For any institution $\mathcal{I}, \mathcal{I}^{\text {pres }}$ is indeed an institution.

The following result is one of the most used institution-theoretic results in formal specification theory laying the foundations for the modern modularization mechanisms; it is also needed for our current paper. It was first formulated and proved in (Goguen and Burstall 1992) (see also (Diaconescu 2008)).

Proposition 2.1 (Colimits of presentations). $\mathcal{I}^{\text {pres }}$ has whatever colimits of signatures $\mathcal{I}$ has.

2.2.3. The method of diagrams The method of diagrams is one of the most important model theoretic methods. In the form presented here it has been introduced at the level of institutionindependent model theory in (Diaconescu 2004a) as a categorical property which formalizes the idea that the class of model homomorphisms from a model $M$ can be represented (by a natural isomorphism) as a class of models of a theory in a signature extending the original signature with syntactic entities determined by $M$. This can be seen as a coherence property between the semantic structure and the syntactic structure of the institution. By following the basic principle 
that a structure is rather defined by its homomorphisms (arrows) than by its objects, the semantical structure of an institution is given by its model homomorphisms. On the other hand the syntactical structure of an institution is essentially determined by its atomic sentences.

The institution-independent method of diagrams pervades the development of a lot of model theoretic results, many of these being presented in (Diaconescu 2008). In the context of our current work, the general concept of diagram allows for a smooth definition for the semantics of predefined types. This role was clearly recognized right from the seminal work (Goguen and Meseguer 1987), but there of course within the concrete framework of Horn clause logic with equality.

Definition 2.5 (The method of diagrams). An institution $\mathcal{I}$ has diagrams when for each signature $\Sigma$ and each $\Sigma$-model $M$, there exists a signature $\Sigma_{M}$ and a signature morphism $\iota_{\Sigma}(M): \Sigma \rightarrow$ $\Sigma_{M}$, functorial in $\Sigma$ and $M$, and a set $E_{M}$ of $\Sigma_{M}$-sentences such that $\operatorname{Mod}\left(\Sigma_{M}, E_{M}\right)$ and the comma category $M / \operatorname{Mod}(\Sigma)$ are naturally isomorphic, i.e. the following diagram commutes by the isomorphism $i_{\Sigma, M}$ that is natural in $\Sigma$ and $M$

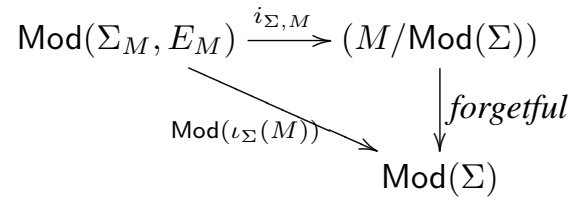

The signature morphism $\iota_{\Sigma}(M): \Sigma \rightarrow \Sigma_{M}$ is called the elementary extension of $\Sigma$ via $M$ and the set $E_{M}$ of $\Sigma_{M}$-sentences is called the diagram of the model $M$. Note that $i_{\Sigma, M}^{-1}\left(1_{M}\right)$ is the initial model of $\left(\Sigma_{M}, E_{M}\right)$, which we denote as $M_{M}$.

The functoriality of $\iota$ means that for each signature morphism $\varphi: \Sigma \rightarrow \Sigma^{\prime}$ and each $\Sigma$-model homomorphism $h: M \rightarrow M^{\prime} \uparrow_{\varphi}$, there exists a presentation morphism $\iota_{\varphi}(h):\left(\Sigma_{M}, E_{M}\right) \rightarrow$ $\left(\Sigma_{M^{\prime}}^{\prime}, E_{M^{\prime}}\right)$ such that

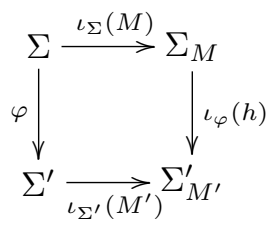

commutes, that $\iota_{\varphi}(h) ; \iota_{\varphi^{\prime}}\left(h^{\prime}\right)=\iota_{\varphi ; \varphi^{\prime}}\left(h ; h^{\prime} \uparrow_{\varphi}\right)$ (for another $\varphi: \Sigma^{\prime} \rightarrow \Sigma^{\prime \prime}$ and $\Sigma^{\prime}$-model homomorphism $\left.h^{\prime}: M^{\prime} \rightarrow M^{\prime \prime} \uparrow \varphi^{\prime}\right)$, and that $\iota_{1_{\Sigma}}\left(1_{M}\right)=1_{\Sigma_{M}}$.

The naturality of $i$ means that for each signature morphism $\varphi: \Sigma \rightarrow \Sigma^{\prime}$ and each $\Sigma$-model homomorphism $h: M \rightarrow M^{\prime} \uparrow_{\varphi}$ the following diagram commutes:

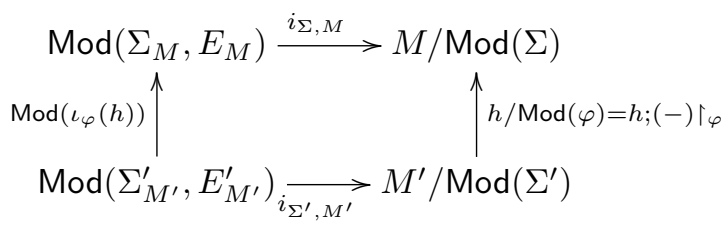

Example 2.3. In $F O L$, for any $(S, F, P)$-model $M$, let $\left(F_{M}\right)_{\rightarrow s}=F_{\rightarrow s} \cup M_{s}$, otherwise let $\left(F_{M}\right)_{w \rightarrow s}=F_{w \rightarrow s}$, and let $M_{M}$ be the $\left(S, F_{M}, P\right)$-expansion of $M$ such that $M_{m}=m$ for each $m \in M$. Then $(S, F, P) \hookrightarrow\left(S, F_{M}, P\right)$ is the elementary extension of $M$. For any signature 
morphism $\varphi:(S, F, P) \rightarrow\left(S^{\prime}, F^{\prime}, P^{\prime}\right)$ and any $(S, F, P)$-model homomorphism $h: M \rightarrow$ $\left.M^{\prime}\right|_{\varphi}$, the signature morphism $\iota_{\varphi}(h):\left(S, F_{M}, P\right) \rightarrow\left(S^{\prime}, F_{M^{\prime}}^{\prime}, P^{\prime}\right)$ is defined by

$-\iota_{\varphi}(h)^{\mathrm{st}}=\varphi^{\mathrm{st}}$ and $\iota_{\varphi}(h)^{\mathrm{rl}}=\varphi^{\mathrm{rl}}$, and

- $\iota_{\varphi}(h)^{\mathrm{op}}(\sigma)= \begin{cases}\varphi^{\mathrm{op}}(\sigma) & \text { when } \sigma \in F_{w \rightarrow s} \text { for any arity } w \text { and any sort } s, \\ h_{s}(\sigma) & \text { when } \sigma \in M_{s} \text { for any sort } s .\end{cases}$

Depending on the chosen concept of model homomorphism one may get various concepts of diagrams as follows:

1 For the ordinary homomorphisms, the diagram $E_{M}$ is the set of all (relational or equational) atoms satisfied by $M_{M}$.

2 When one restricts homomorphisms to injective ones, $E_{M}$ consists of all atoms and negations of atomic equations satisfied by $M_{M}$.

3 When one restricts homomorphisms to the closed ones ( $h: M \rightarrow N$ is closed $^{\ddagger}$ if $M_{\pi}=$ $h^{-1}\left(N_{\pi}\right)$ for each $\left.\pi \in P\right), E_{M}$ consists of all atoms and negations of atomic relations satisfied by $M_{M}$.

4 When one restricts homomorphisms to closed injective model homomorphisms, $E_{M}$ consists of all atoms and all negations of atoms satisfied by $M_{M}$.

5 When model homomorphisms are restricted to elementary embeddings (according to the literature, e.g. (Chang and Keisler 1990), $h: M \rightarrow N$ is elementary embedding when $M_{M}$ and $N_{h}$ satisfy the same set of sentences, where $N_{h}$ is the expansion of $N$ such that $\left(N_{h}\right)_{m}=h(m)$ for each $\left.m \in|M|\right), E_{M}=\left\{\rho \mid M_{M} \models \rho\right\}$.

Moreover it is easy to note that $H C L$ has the same diagrams as $F O L$ with the standard model homomorphisms. This is due to the fact that the diagrams $E_{M}$ in $F O L$ consist of atoms only, and atoms qualify as $H C L$ sentences too.

2.2.4. Model amalgamation The crucial role of model amalgamation for the semantics studies of formal specifications comes up in very many works in the area, a few early examples being (Sannella and Tarlecki 1988; Tarlecki 1986b; Meseguer 1989; Diaconescu et al. 1993). The model amalgamation property is a necessary condition in many institution-independent model theoretic results (see (Diaconescu 2008)), thus being one of the most desirable properties for an institution. It can be considered even as more fundamental than the satisfaction condition since in institutions with quantifications it is used in one of its weak forms in the proof of the satisfaction condition at the induction step corresponding to quantifiers.

Model amalgamation properties for institutions formalize the possibility of amalgamating models of different signatures when they are consistent on some kind of generalized 'intersection' of signatures.

Definition 2.6 (Model amalgamation). A commutative square of signature morphisms

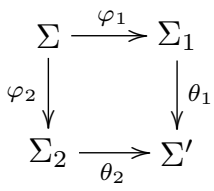

\footnotetext{
$\ddagger$ Other works may call this kind of model homomorphisms strong.
} 
is an amalgamation square if and only if for each $\Sigma_{1}$-model $M_{1}$ and a $\Sigma_{2}$-model $M_{2}$ such that $M_{1} \uparrow_{\varphi_{1}}=M_{2} \uparrow_{\varphi_{2}}$, there exists an unique $\Sigma^{\prime}$-model $M^{\prime}$, denoted $M_{1} \otimes_{\varphi_{1}, \varphi_{2}} M_{2}$, or $M_{1} \otimes M_{2}$ for short when there is no danger of ambiguity, such that $M^{\prime} \uparrow_{\theta_{1}}=M_{1}$ and $M^{\prime} \uparrow_{\theta_{2}}=M_{2}$.

In most of the institutions formalizing conventional or non-conventional logics, pushout squares of signature morphisms are model amalgamation squares (Diaconescu et al. 1993; Diaconescu 2008). These include our benchmark examples FOL and $H C L$.

Definition 2.7. An institution has model amalgamation when each pushout square of signatures is an amalgamation square.

The following result will be used later in our paper.

Proposition 2.2. Consider the following commutative diagram of signature morphisms.

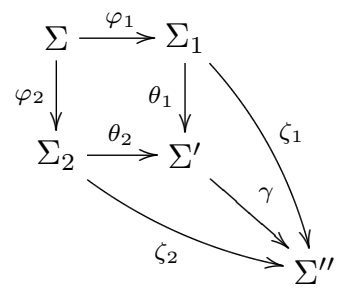

If both the inner square $\left[\varphi_{1}, \varphi_{2}, \theta_{1}, \theta_{2}\right]$ and the outer square $\left[\varphi_{1}, \varphi_{2}, \zeta_{1}, \zeta_{2}\right]$ of this diagram have the model amalgamation property then the mediating signature morphism $\gamma$ is conservative.

Proof. Let $M^{\prime}$ be any $\Sigma^{\prime}$-model. By the model amalgamation property of the outer square $\left[\varphi_{1}, \varphi_{2}, \zeta_{1}, \zeta_{2}\right]$ there exists an unique $\Sigma^{\prime \prime}$-model $M^{\prime \prime}$ such that $M^{\prime \prime} \uparrow_{\zeta_{i}}=M^{\prime} \uparrow_{\theta_{i}}$ for $i \in\{1,2\}$. Let us prove that $M^{\prime \prime}$ is a $\gamma$-expansion of $M^{\prime}$. For this we have just to note that for $i \in\{1,2\}$ we have that $\left(M^{\prime \prime} \uparrow_{\gamma}\right) \uparrow_{\theta_{i}}=M^{\prime \prime} \uparrow_{\theta_{i} ; \gamma}=M^{\prime \prime} \uparrow_{\zeta_{i}}=M_{\theta_{i}}^{\prime}$. By the uniqueness side of the model amalgamation property for the inner square $\left[\varphi_{1}, \varphi_{2}, \theta_{1}, \theta_{2}\right]$ we have that $M^{\prime \prime} \uparrow_{\gamma}=M^{\prime}$.

The following result, also used later in our paper, is part of the folklore of institution theory (a proof can be found in (Diaconescu 2008)).

Proposition 2.3. For any institution $\mathcal{I}$ with model amalgamation, the institution $\mathcal{I}^{\text {pres }}$ of the $\mathcal{I}$-presentations has model amalgamation also.

2.2.5. Liberality Liberality properties express the idea of free constructions at the general institutionindependent level and have been formulated for the first time in the seminal paper (Goguen and Burstall 1992). Historically (Goguen and Burstall 1992) they constitute a general abstract formulation of the formal specification paradigm of 'initial algebra semantics' (Goguen et al. 1977).

Definition 2.8. A signature morphism $\varphi: \Sigma \rightarrow \Sigma^{\prime}$ in an arbitrary institution is liberal if the corresponding model functor $\operatorname{Mod}(\varphi)$ has a left adjoint, i.e. for each $\Sigma$-model $M$ there exists a 
$\Sigma^{\prime}$-model $M^{\varphi}$ and a $\Sigma$-model homomorphism $\eta_{M}: M \rightarrow\left(M^{\varphi}\right) \uparrow_{\varphi}$

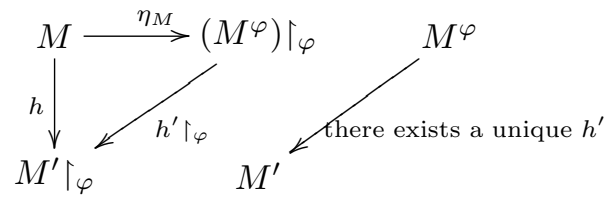

such that for each $\Sigma^{\prime}$-model $M^{\prime}$ and for each $\Sigma$-model homomorphism $h: M \rightarrow M^{\prime}{ }^{{ }_{\varphi}}$, there exists a unique $\Sigma^{\prime}$-model homomorphism $h^{\prime}: M^{\varphi} \rightarrow M^{\prime}$ such that $\eta_{M} ; h^{\prime} \uparrow_{\varphi}=h$.

Liberality of all signature morphisms in institutions is a rather common property, exceptions are rare. A set of widely applicable (including our benchmark examples $F O L$ and $H C L$ ) sufficient conditions for liberality have been developed in (Tarlecki 1986b; Diaconescu 2004a) (see also (Diaconescu 2008)).

\subsection{Interpolation}

In the algebraic specification literature there are several institution-independent formulations of interpolation, all of them being strongly related. For example (Tarlecki 1986a) is one of the first works introducing the concept of interpolation at the level of abstract institutions. The common feature of these formulations is that they generalise the conventional intersection-union (of signatures) framework to commutative squares of signature morphisms. In most cases these commutative squares are required to be pushouts (like in (Tarlecki 1986b; Borzyszkowski 2000; Borzyszkowski 2001; Dimitrakos and Maibaum 2000)), in other case the signature morphisms are required to be (abstract) inclusions (like in (Diaconescu et al. 1993)). However in (Diaconescu 2004c) it has been noticed that the mere formulation of interpolation does not require any extra technical assumptions besides a commuting square of signature morphisms, the role of such additional assumptions having more to do with the proof of interpolation properties rather than with its formulation.

Here we review the concept of institution-independent interpolation and develop a couple of new general results which we need for our current work.

Definition 2.9 (Craig-Robinson interpolation). In any institution we say that a commutative square of signature morphisms

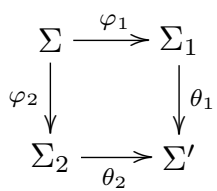

is a Craig-Robinson Interpolation square (abbreviated $C R I$ square) when for each set $E_{1}$ of $\Sigma_{1}$ sentences and each sets $E_{2}$ and $\Gamma_{2}$ of $\Sigma_{2}$-sentences, if $\theta_{1}\left(E_{1}\right) \cup \theta_{2}\left(\Gamma_{2}\right) \models_{\Sigma^{\prime}} \theta_{2}\left(E_{2}\right)$, then there exists a set $E$ of $\Sigma$-sentences such that $E_{1} \models_{\Sigma_{1}} \varphi_{1}(E)$ and $\Gamma_{2} \cup \varphi_{2}(E) \models_{\Sigma_{2}} E_{2}$.

The particular case of Craig-Robinson interpolation when $\Gamma_{2}$ is empty is called Craig interpolation and in logic this case is usually more studied than Craig-Robinson interpolation. CraigRobinson form of interpolation seems to have been first introduced in first order logic by (Mae- 
hara 1961). Several works (Bergstra et al. 1990; Diaconescu et al. 1993; Dimitrakos and Maibaum 2000; Diaconescu 2008) show that Craig-Robinson rather than Craig may be the appropriate interpolation concept for formal specification studies. A particular important example in this sense is given by Borzyszkowski's complete calculus for structured specifications of (Borzyszkowski 2000) which in reality relies upon the Craig-Robinson form of interpolation (this was shown in (Diaconescu 2008) which corrects the rather restricted original result of (Borzyszkowski 2000) relying upon Craig interpolation plus additional conditions for the base institutions, the latter narrowing significantly the range of the applications of this important and beautiful result). Moreover even in model theory sometimes (Petria and Diaconescu 2006) Craig-Robinson seems to be the appropriate form of interpolation. This is one of the reasons we adopt here this form of interpolation, another one being just technical. The name "Craig-Robinson" has been used for instances of the corresponding interpolation property in (Shoenfield 1967; Veloso 1996; Dimitrakos and Maibaum 2000), "Maehara interpolation" in sentential logic studies, while "strong Craig interpolation" has been used in (Diaconescu et al. 1993). We mention that Craig-Robinson and Craig forms of interpolation can be shown equivalent under some additional conditions on the institution (Diaconescu 2008). For example this applies to FOL (which perhaps is the main reason why in conventional logic Craig-Robinson formulation of interpolation is shadowed by the simpler Craig formulation), but not to $H C L$.

Another important aspect of Def. 2.9 is that it uses sets of sentences rather than single sentences, as is common in conventional logic. The works (Rodenburg 1991) and (Diaconescu et al. 1993) argue succesfully that the formulation of interpolation in terms of sets of sentences is more natural than the more traditional formulations in terms of single sentences. First, on the one hand, the applications of interpolation do not require the single sentence formulation, and on the other hand the single sentence formulation excludes important examples such as equational or Horn logics. Then, in traditional works on or using interpolation, under an assumption of compactness the two formulations can be shown equivalent (Diaconescu 2008).

The definition below formulates interpolation as a property of institutions. In its current form it has been introduced in (Diaconescu 2004d) as a simplified variant of the original definition of (Borzyszkowski 2000).

Definition 2.10 $(\langle\mathcal{L}, \mathcal{R}\rangle$-interpolation). For any classes of signature morphisms $\mathcal{L}, \mathcal{R} \subseteq$ Sig in any institution, we say that the institution has the Craig-Robinson $\langle\mathcal{L}, \mathcal{R}\rangle$-interpolation if each pushout square of signature morphisms of the form

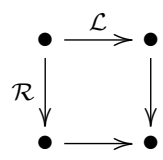

is a CRI square.

Example 2.4. According to (Găină and Popescu 2004; Borzyszkowski 2005), FOL has CraigRobinson $\left\langle\mathbb{S} i g^{F O L},(i * *)\right\rangle$ - and $\left\langle(i * *)\right.$, Sig $\left.{ }^{F O L}\right\rangle$-interpolation, where $(i * *)$ is the class of the $F O L$-signature morphisms that are injective on the sort symbols. Interestingly, this result which stayed as a conjecture for several years has received an elegant proof in (Găină and Popescu 2004) 
using an institution-independent method; in fact the result proved there is institution-independent and thus much more general than $F O L$ interpolation.

Example 2.5. According to (Diaconescu 2008), $H C L$ has Craig-Robinson $\left\langle(i e *)\right.$, $\left.\mathbb{S} i g^{H C L}\right\rangle$ interpolation where $(i e *)$ is the class of $H C L$-signature morphisms that are injective on the sorts and such that no operation symbol outside the image of the signature morphism is allowed to have the sort in the image of the signature morphism (in other words if $\varphi:(S, F, P) \rightarrow\left(S^{\prime}, F^{\prime}, P^{\prime}\right)$ and $\sigma^{\prime} \in F_{w^{\prime} \rightarrow s^{\prime}}^{\prime}$ with $s^{\prime} \in \varphi(s)$ then there exists $\sigma \in F_{w \rightarrow s}$ such that $\left.\varphi(\sigma)=\sigma^{\prime}\right)$. The proof of this result involves the interpolation result for Grothendieck institutions of (Diaconescu 2004d).

Below in the paper we will make use of the following result.

Proposition 2.4. Consider the following commutative diagram of signature morphisms.

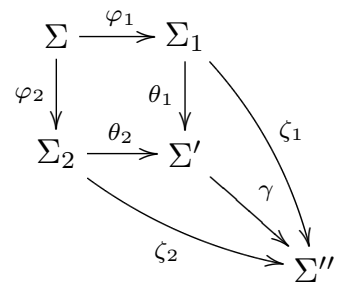

If the inner square $\left[\varphi_{1}, \varphi_{2}, \theta_{1}, \theta_{2}\right]$ is a CRI square and the mediating signature morphism $\gamma$ is conservative then the outer square $\left[\varphi_{1}, \varphi_{2}, \zeta_{1}, \zeta_{2}\right]$ is a CRI square too.

Proof. Let $E_{1} \subseteq \operatorname{Sen}\left(\Sigma_{1}\right)$ and $E_{2}, \Gamma_{2} \subseteq \operatorname{Sen}\left(\Sigma_{2}\right)$ such that

$$
\zeta_{1}\left(E_{1}\right) \cup \zeta_{2}\left(\Gamma_{2}\right) \models_{\Sigma^{\prime \prime}} \zeta_{2}\left(E_{2}\right)
$$

which means

$$
\gamma\left(\theta_{1}\left(E_{1}\right) \cup \theta_{2}\left(\Gamma_{2}\right)\right) \models_{\Sigma^{\prime \prime}} \gamma\left(\theta_{2}\left(E_{2}\right)\right) .
$$

Because $\gamma$ is conservative, by Fact 2.1 we have that

$$
\theta_{1}\left(E_{1}\right) \cup \theta_{2}\left(\Gamma_{2}\right) \models \Sigma_{\Sigma^{\prime}} \theta_{2}\left(E_{2}\right) .
$$

Because the inner square $\left[\varphi_{1}, \varphi_{2}, \theta_{1}, \theta_{2}\right]$ is a CRI square there exists an interpolant $E \subseteq \operatorname{Sen}(\Sigma)$ such that $E_{1} \models_{\Sigma_{1}} \varphi_{1}(E)$ and $\varphi_{2}(E) \cup \Gamma_{2} \models_{\Sigma_{2}} E_{2}$.

The last part of this section is devoted to the lifting of interpolation properties from a base institution to the institution of its presentations. This result, used below for the proof of the main result of the paper, requires the Craig-Robinson formulation of interpolation as it cannot be developed for Craig interpolation.

Notation 2.2. For any institution $\mathcal{I}$ and a class $\mathcal{S} \subseteq \mathbb{S} i g$ of signature morphisms let $\mathcal{S}^{\text {pres }}$ be the class of presentation morphisms $\varphi$ such that $\varphi \in \mathcal{S}$ (as signature morphism).

Proposition 2.5. The institution $\mathcal{I}^{\text {pres }}$ of the presentations of $\mathcal{I}$ has the Craig-Robinson $\left\langle\mathcal{L}^{\text {pres }}, \mathcal{R}^{\text {pres }}\right\rangle$ interpolation if $\mathcal{I}$ has the Craig-Robinson $\langle\mathcal{L}, \mathcal{R}\rangle$-interpolation. 
Proof. Consider a pushout of presentations

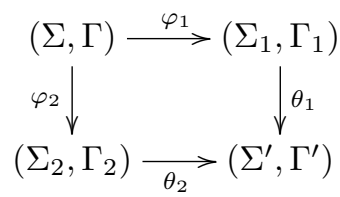

such that $\varphi_{1} \in \mathcal{L}, \varphi_{2} \in \mathcal{R}$ and let $E_{1} \subseteq \operatorname{Sen}\left(\Sigma_{1}\right)$ and $E_{2}, K_{2} \in \operatorname{Sen}\left(\Sigma_{2}\right)$ such that $\theta_{1}\left(E_{1}\right) \cup$ $\theta_{2}\left(K_{2}\right) \models{ }_{\left(\Sigma^{\prime}, \Gamma^{\prime}\right)} \theta_{2}\left(E_{2}\right)$.

By the general construction of colimits of presentations (see (Goguen and Burstall 1992; Diaconescu 2008)) $\Gamma^{\prime} \models \theta_{1}\left(\Gamma_{1}\right) \cup \theta_{2}\left(\Gamma_{2}\right)$ and $\left[\varphi_{1}, \varphi_{2}, \theta_{1}, \theta_{2}\right]$ form a pushout square in $\mathbb{S i g}$. Therefore $\theta_{1}\left(E_{1} \cup \Gamma_{1}\right) \cup \theta_{2}\left(K_{2} \cup \Gamma_{2}\right)==_{\Sigma^{\prime}} \theta_{2}\left(E_{2}\right)$. By the CRI property for the base institution $\mathcal{I}$, there exists an interpolant $E \subseteq \operatorname{Sen}(\Sigma)$ such that

$$
E_{1} \cup \Gamma_{1} \models \Sigma_{\Sigma_{1}} \varphi_{1}(E) \text { and } \varphi_{2}(E) \cup K_{2} \cup \Gamma_{2} \models_{\Sigma_{2}} E_{2}
$$

But these just mean

$$
E_{1} \models_{\left(\Sigma_{1}, \Gamma_{1}\right)} \varphi_{1}(E) \text { and }\left.\varphi_{2}(E) \cup K_{2}\right|_{\left(\Sigma_{2}, \Gamma_{2}\right)} E_{2}
$$

which shows the CRI property for presentations.

\section{Institutions of predefined types}

In this section we introduce our updated institution-independent semantics for predefined types. The section is structured as follows:

1 A rather detailed presentation of an example with the aim to help the understanding of the abstract semantics introduced in this section.

2 The definition of the institution of the predefined types, including the proof of its satisfaction condition.

3 The proof of the model amalgamation property for the institutions of predefined types.

\subsection{The Euclidean plane}

Let us consider a specification of the Euclidean plane $\mathbb{R}^{2}$ as a vector space. For this we use two sorts, Real for the real numbers as scalars, and Vect for the vectors. Let $S=\{$ Real, Vect $\}$. We also need operations, denoted $F$, which are the usual ring operations on the scalar sort Real, i.e. $F_{\rightarrow \text { Real }}=\{0\}, F_{\text {Real } \rightarrow \text { Real }}=\left\{-_{-}\right\}$, and $F_{\text {RealReal } \rightarrow \text { Real }}=\left\{-_{-}+_{-}, *_{-}\right\}$, plus the following operations for the vector sort Vect: $F_{\rightarrow \text { Vect }}=\{0\}, F_{\text {RealReal } \rightarrow \text { vect }}=\left\{\left\langle\left\langle_{-},\right\rangle\right\}, F_{\text {Vect } \rightarrow \text { vect }}=\left\{-_{-}\right\}\right.$, $F_{\text {VectVect } \rightarrow \text { Vect }}=\left\{{ }_{-}+_{-}\right\}$, and $F_{\text {RealVect } \rightarrow \text { Vect }}=\left\{{ }_{-} *_{-}\right\}$. Moreover, in addition to the operations $F$ listed above we have to consider also the real numbers. For reasons that will become more transparent below, these have to be considered not only as purely syntactic entities, but together with their model theoretic structure. This yields a pair $((S, F), \mathbb{R})$ with $\mathbb{R}$ denoting the model (ring) of the real numbers, which defines a signature-like framework for specifiying the Euclidean plane. It is not a signature in the conventional concrete sense since besides the conventional syntactic entities (which appear as $F O L$ signature $(S, F)$, so without relation symbols) it also contains the 
model (ring) $\mathbb{R}$ of the real numbers. However, form the abstract viewpoint of institution theory which regards signatures as abstract objects in categories, the pair $((S, F), \mathbb{R})$ may play the role of a signature.

Now let us consider the models for such signature, models which should include the Euclidean plane $\mathbb{R}^{2}$. These models have to contain an interpretation of the real numbers, including their ring structure. This is motivated by the fact that besides the interpretations of the actual numbers considered as constants, one has also to consider all the relationships between them given by their ring structure. For example, (the interpretation of) $1.37 * 5.23$ should be equal to (the interpretation of) 7.1651 in all such models. So, how can we interpret the reals inside the models of $(S, F)$ ? The answer is simple: by considering ring homomorphisms from $\mathbb{R}$ to the relevant sub-part of the respective model given by the sub-signature of the ring of the real numbers. In other words, a model for $((S, F), \mathbb{R})$ is just a ring homomorphism $\mathbb{R} \rightarrow M{\mid \Sigma_{0}}_{\text {w }}$ where $M$ is any $(S, F)$-model and $\Sigma_{0}$ is the sub-signature of $(S, F)$ containing only the entities which refer to the sort Real. Now how do we characterize the Euclidean plane $\mathbb{R}^{2}$ among these models? We need to consider only those models that satisfy the axioms of a two-dimensional vector space:

$$
\begin{aligned}
& 0=\langle 0,0\rangle, \\
& \left(\forall\left\{a, b, a^{\prime}, b^{\prime}: \operatorname{Real}\right\}\right)\langle a, b\rangle+\left\langle a^{\prime}, b^{\prime}\right\rangle=\left\langle a+a^{\prime}, b+b^{\prime}\right\rangle, \\
& (\forall\{k, a, b: \operatorname{Real}\}) k *\langle a, b\rangle=\langle k * a, k * b\rangle, \\
& (\forall\{a, b: \operatorname{Real}\})-\langle a, b\rangle=\langle-a,-b\rangle .
\end{aligned}
$$

But this is not enough since among these models we may still find models quite different from $\mathbb{R}^{2}$. For example a model interpreting Vect as the set of the real numbers and $\left\langle_{-},{ }_{-}\right\rangle$as addition still satisfies the above axioms of a vector space. The answer to this problem is that $\mathbb{R}^{2}$ is the initial model of the above specification.

Let us sum up the situation so far for this example:

- The signature consists of

- the $F O L$ signature $(S, F)$,

- the sub-signature $\Sigma_{0}$ of $(S, F)$, and

- the ring $\mathbb{R}$ of the real numbers as a $\Sigma_{0}$-model.

- A model consists of

- an $(S, F)$-model, and

- a $\Sigma_{0}$-model homomorphism $\mathbb{R} \rightarrow M \Gamma_{\Sigma_{0}}$.

This quite complex situation, which is characteristic to previous works such as (Diaconescu 2000), can be simplified quite a lot mathematically while maintaining the content, by replacing the sub-signature $\Sigma_{0}$ and the model $\mathbb{R}$ by $\mathbb{R}^{\prime}$, the free $(S, F)$-model over $\mathbb{R}$. The model $\mathbb{R}^{\prime}$ exists because all signature morphisms in FOL are liberal (see (Diaconescu 2008)). The main point behind this simplification is the freeness of $\mathbb{R}^{\prime}$, which guarantees a natural bijection between the homomorphisms $\mathbb{R} \rightarrow M \Gamma_{\Sigma_{0}}$ and the homomorphisms $\mathbb{R}^{\prime} \rightarrow M$. We may sum up the new situation of the Euclidean plane specification as follows:

- the signature is just the pair $\left((S, F), \mathbb{R}^{\prime}\right)$ consisting of a $F O L$ signature and a model of this signature, and

- the category of the models of $\left((S, F), \mathbb{R}^{\prime}\right)$ is just the comma category $\mathbb{R}^{\prime} / \operatorname{Mod}^{F O L}(S, F)$, and the Euclidean plane $\mathbb{R}^{2}$ is the initial model satisfying the four axioms above. 
We have already invoked rather informally the concepts of sentence and satisfaction for the signature of the Euclidean plane specification, now we introduce them formally. As expected, the sentences are just $F O L$ sentences but using terms formed from the operations from $F$ plus all the real numbers regarded as (syntactic) constants; let us denote this signature by $\left(S, F_{\mathbb{R}}\right)$. For example $(\exists X, Y) \pi *\langle X, Y\rangle=\langle 1.2,2.3\rangle$ is such a sentence. We now argue that that using $\left(S, F_{\mathbb{R}}\right)$-sentences is the same with using $\left(S, F_{\mathbb{R}^{\prime}}\right)$-sentences, where $\left(S, F_{\mathbb{R}^{\prime}}\right)$ is the elementary extension of $(S, F)$ via $\mathbb{R}^{\prime}$. In order to understand this fact, it is enough to note that, on the one hand each $\left(S, F_{\mathbb{R}}\right)$-term is obviously a $\left(S, F_{\mathbb{R}^{\prime}}\right)$-term, and on the other hand there are maps $p$ from $\left(S, F_{\mathbb{R}^{\prime}}\right)$-terms to $\left(S, F_{\mathbb{R}}\right)$-terms which preserve the interpretation of the terms in the models. In order to describe the mapping $p$, let us recall that the elements of $\mathbb{R}^{\prime}$ are the equivalence classes of $\left(S, F_{\mathbb{R}}\right)$-terms under the congruence $\sim$ generated by the diagram of $\mathbb{R}$ (i.e. all equalities between terms formed with real numbers and the ring operations, such as $1.2 * 1.1=1.32$ for example). Then $p$ can be defined by $p(t / \sim)=t$ for any $t / \sim \in \mathbb{R}^{\prime}$. Of course there can be several such maps depending on the choice of representatives from the equivalence classes; our argument is invariant with respect to such choice. We can now formulate our conclusion about the sentences:

the $\left((S, F), \mathbb{R}^{\prime}\right)$-sentences are precisely the $F O L\left(S, F_{\mathbb{R}^{\prime}}\right)$-sentences, where $\left(S, F_{\mathbb{R}^{\prime}}\right)$ is the elementary extension of $(S, F)$ via $\mathbb{R}^{\prime}$.

The satisfaction of a $\left((S, F), \mathbb{R}^{\prime}\right)$-sentence $\rho$ by a $\left((S, F), \mathbb{R}^{\prime}\right)$-model $h: \mathbb{R}^{\prime} \rightarrow M$ is given by the FOL satisfaction relation by interpreting any constant denoting a real number $r$ by its corresponding image $h(r)$ in $M$. This can be formally defined as the satisfaction of $\rho$, regarded as a $F O L\left((S, F), \mathbb{R}^{\prime}\right)$-sentence, by the correspondent of $h$ to a model of the diagram of $\mathbb{R}^{\prime}$ through the natural isomorphism between $\mathbb{R}^{\prime} / \operatorname{Mod}^{F O L}(S, F)$ and $\operatorname{Mod}^{F O L}\left(\left(S, F_{\mathbb{R}^{\prime}}\right), E_{\mathbb{R}^{\prime}}\right)$.

\subsection{The definition of the institution of predefined types}

The following definition collects the conclusions of the Euclidean plane example above in a form of a general definition.

Definition 3.1. Let $\mathcal{I}=(\mathbb{S} i g$, Sen, Mod, $\models, \iota)$ be any institution with diagrams. The institution with predefined types over $\mathcal{I}$, denoted $\mathcal{I}^{2}=\left(\mathbb{S}^{2} g^{2}, \operatorname{Sen}^{2}, \operatorname{Mod}^{2}, \models^{2}\right)$, is defined as follows:

- the category of signatures with predefined type $\mathbb{S}^{2} g^{2}$ is the Grothendieck category Mod ${ }^{\sharp}$,

- $\operatorname{Sen}^{2}(\Sigma, A)=\operatorname{Sen}\left(\Sigma_{A}\right)$ for each signature $(\Sigma, A)$, and $\operatorname{Sen}^{2}(\varphi, h)=\operatorname{Sen}\left(\iota_{\varphi}(h)\right)$ for each signature morphism $(\varphi, h)$,

- $\operatorname{Mod}^{2}(\Sigma, A)=A / \operatorname{Mod}(\Sigma)$ for each signature $(\Sigma, A)$, and

- for each signature $(\Sigma, A),(h: A \rightarrow M) \models_{(\Sigma, A)}^{2} \rho$ if and only if $i_{\Sigma, A}^{-1}(h) \models_{\Sigma_{A}} \rho$.

Proposition 3.1. $\mathcal{I}^{\prime}$ is an institution indeed.

Proof. We have to prove the satisfaction condition for $\mathcal{I}^{2}$. For this we consider a $\mathcal{I}^{\prime}$ signature morphism $(\varphi, h):(\Sigma, A) \rightarrow\left(\Sigma^{\prime}, A^{\prime}\right)$, a $\left(\Sigma^{\prime}, A^{\prime}\right)$-model $f^{\prime}: A^{\prime} \rightarrow M^{\prime}$ and a $(\Sigma, A)$-sentence $\rho$. We have that 


$$
\begin{array}{rll}
f^{\prime} \uparrow_{(\varphi, h)} \models_{(\Sigma, A)}^{2} \rho & \text { iff } \quad\left(h ; f^{\prime} \uparrow_{\varphi}\right) \models_{(\Sigma, A)}^{2} \rho & \text { (by the definition of reducts in } \mathcal{I}^{\imath} \text { ) } \\
& \text { iff } i_{\Sigma, A}^{-1}\left(h ; f^{\prime} \uparrow_{\varphi}\right) \models_{\Sigma_{A}} \rho & \text { (by the definition of } \models^{2} \text { ) } \\
& \text { iff } i_{\Sigma^{\prime}, A^{\prime}}^{-1}\left(f^{\prime}\right) \uparrow_{\iota_{\varphi}(h) \models_{\Sigma_{A}} \rho} \text { (by the naturality of } i \text { in } \Sigma \text { and } A \text { ) } \\
& \text { iff } i_{\Sigma^{\prime}, A^{\prime}}^{-1}\left(f^{\prime}\right) \models_{\Sigma_{A^{\prime}}^{\prime}} \iota_{\varphi}(h)(\rho) & \text { (by the satisfaction condition in } \mathcal{I} \text { ) } \\
& \text { iff } f^{\prime} \models_{\left(\Sigma^{\prime}, A^{\prime}\right)}^{2}(\varphi, h)(\rho) & \text { (by the definition of }=^{2} \text { ). }
\end{array}
$$

The following property will be used below in the paper.

Fact 3.1. In any institution with diagrams ( $\operatorname{Sig}$, Sen, Mod, $\models, \iota)$, for any signature $\Sigma$ and any $\Sigma$-model $A$, for any sets $E$ and $\Gamma$ of $\Sigma_{A}$-sentences

$$
E \models_{(\Sigma, A)}^{2} \Gamma \text { if and only if } E \models_{\left(\Sigma_{A}, E_{A}\right)}^{\text {pres }} \Gamma \text {. }
$$

\subsection{The model amalgamation property for the institutions of predefined types}

Model amalgamation is a very useful property of institutions, often a prerequisite for interpolation (Diaconescu 2004c; Găină and Popescu 2004). Our work is no exception, the following result being necessary for the proof of our main interpolation result.

Proposition 3.2. For any base institution with diagrams $\mathcal{I}=(\mathbb{S i g}, \operatorname{Sen}, \operatorname{Mod}, \models, \iota)$ such that

1 its category of signatures $\mathbb{S i g}$ has pushouts,

2 each of its signature morphisms is liberal, and

3 for each signature $\Sigma \in|\operatorname{Sig}|$, the category $\operatorname{Mod}(\Sigma)$ of $\Sigma$-models has pushouts,

the corresponding institution of predefined types $\mathcal{I}^{2}$ has pushouts of signatures. In addition, if the base institution $\mathcal{I}$ has model amalgamation, then $\mathcal{I}^{l}$ has model amalgamation too.

Proof. For the first part of the proposition we have only to note that the category $\mathbb{S i g}^{2}$ of the signatures of $\mathcal{I}^{2}$ is the Grothendieck category determined by the model functor Mod: Sig $^{\text {op }} \rightarrow$ $\mathbb{C}$ at regarded as an indexed category. That $\mathbb{S i g}^{2}$ has pushouts follows from the general result of existence of colimits in Grothendieck categories (see (Tarlecki et al. 1991), for example).

For the second part of the proposition we consider a pushout in $\mathbb{S} i g^{2}$ as shown in the diagram below.

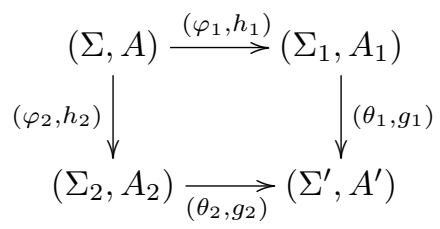

Let $f_{1}: A_{1} \rightarrow M_{1}$ and $f_{2}: A_{2} \rightarrow M_{2}$ such that $h_{1} ; f_{1} \uparrow_{\varphi_{1}}=h_{2} ; f_{2} \uparrow_{\varphi_{2}}$. This also impplies that $M_{1} \uparrow_{\varphi_{1}}=M_{2} \uparrow_{\varphi_{2}}$. From the construction of colimits in Grothendieck categories we have that the following commutative square is a pushout in $\mathbb{S i g}$.

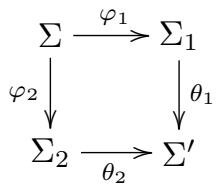


Hence by the model amalgamation hypothesis on $\mathcal{I}$, there exists an unique $\Sigma^{\prime}$-model $M^{\prime}$ such that $M^{\prime} \uparrow_{\theta_{i}}=M_{i}$ for each $i \in\{1,2\}$. Note that $\left(\theta_{i}, f_{i}\right):\left(\Sigma_{i}, A_{i}\right) \rightarrow\left(\Sigma^{\prime}, A^{\prime}\right)$ are morphisms in $\mathbb{S}_{i g}$ and that $\left(\varphi_{1}, h_{1}\right) ;\left(\theta_{1}, f_{1}\right)=\left(\varphi_{2}, h_{2}\right) ;\left(\theta_{2}, f_{2}\right)$.

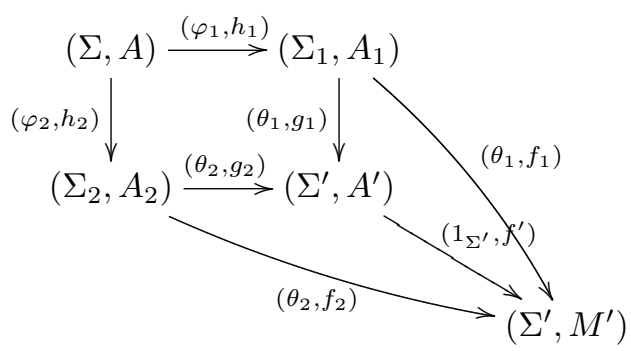

By the pushout property of the square $\left[\left(\varphi_{1}, h_{1}\right),\left(\varphi_{2}, h_{2}\right),\left(\theta_{1}, g_{1}\right),\left(\theta_{2}, g_{2}\right)\right]$ there exists an unique signature morphism $\left(1_{\Sigma^{\prime}}, f^{\prime}\right)$ such that $\left(\theta_{i}, g_{i}\right) ;\left(1_{\Sigma^{\prime}}, f^{\prime}\right)=\left(\theta_{i}, f_{i}\right)$ for $i \in\{1,2\}$, which means that the model homomorphism $f^{\prime}$ is the unique amalgamation of $f_{1}$ and $f_{2}$.

\section{Interpolation for predefined types}

This section develops the main technical result of this paper, namely the institution-independent interpolation theorem for predefined types. It also develops two concrete instances of this result.

The concept of intrpolation for predefined types is obtained immediately by applying the general concept of interpolation (Def. 2.9 and 2.10) to the institution of the predefined types (Def. 3.1).

Notation 4.1. Let $\mathcal{I}=(\mathbb{S} i g$, Sen, Mod, $\mid=, \iota)$ be an institution with diagrams. For any class $\mathcal{S} \subseteq \mathbb{S} i g$ of signature morphisms, let $\mathcal{S}^{2}$ denote the class of the signature morphisms in $\mathcal{I}^{2}$ defined by $\mathcal{S}^{l}=\left\{(\varphi, h) \in \mathbb{S i g}^{2} \mid \iota_{\varphi}(h) \in \mathcal{S}\right\}$.

Theorem 4.1. Let $\mathcal{I}=(\mathbb{S i g}$, Sen, Mod, $\models, \iota)$ be an institution with diagrams such that

1 its category of signatures $\mathbb{S} i g$ has pushouts,

2 each of its signature morphisms is liberal,

3 it has model amalgamation, and

4 for each signature $\Sigma \in|\operatorname{Sig}|$, the category $\operatorname{Mod}(\Sigma)$ of $\Sigma$-models has pushouts.

If $\mathcal{I}$ has Craig-Robinson $\langle\mathcal{L}, \mathcal{R}\rangle$-interpolation then the corresponding institution with predefined types $\mathcal{I}^{2}$ has Craig-Robinson $\left\langle\mathcal{L}^{2}, \mathcal{R}^{2}\right\rangle$-interpolation.

Proof. By Prop. 3.2 we have that $\mathcal{I}^{2}$ has pushouts of signatures. Let us consider a pushout of signature morphisms in $\mathbb{S i g}^{2}$

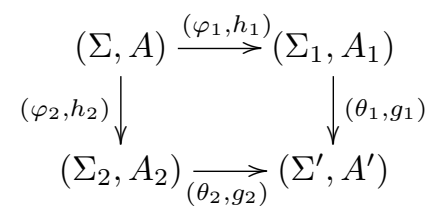

such that $\left(\varphi_{1}, h_{1}\right) \in \mathcal{L}^{2}$ and $\left(\varphi_{2}, h_{2}\right) \in \mathcal{R}^{2}$. 
We consider the following commutative diagram of $\mathcal{I}$-presentation morphisms with the inner square $\left[\iota_{\varphi_{1}}\left(h_{1}\right), \iota_{\varphi_{2}}\left(h_{2}\right), \phi_{1}, \phi_{2}\right]$ being a pushout of presentations and $\gamma$ being the unique mediating presentation morphism such that the diagram commutes.

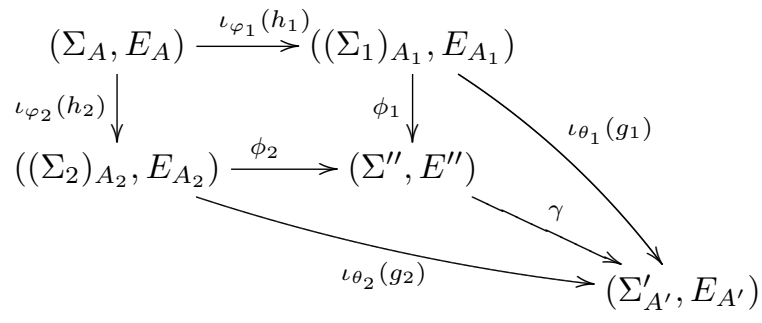

Note that the commutativity of the outer square $\left[\iota_{\varphi_{1}}\left(h_{1}\right), \iota_{\varphi_{2}}\left(h_{2}\right), \iota_{\theta_{1}}\left(g_{1}\right), \iota_{\theta_{2}}\left(g_{2}\right)\right]$ follows directly from the commutativity of the considered pushout square of $\mathcal{I}^{\mathcal{L}}$-signature morphisms since by the functoriality of $\iota$ we have that $\iota_{\varphi_{i}}\left(h_{i}\right) ; \iota_{\theta_{i}}\left(g_{i}\right)=\iota_{\varphi_{i} ; \theta_{i}}\left(h_{i} ; g_{i} \uparrow_{\theta_{i}}\right)$ for $i \in\{1,2\}$.

Since the inner square $\left[\iota_{\varphi_{1}}\left(h_{1}\right), \iota_{\varphi_{2}}\left(h_{2}\right), \phi_{1}, \phi_{2}\right]$ of the diagram is a pushout square of $\mathcal{I}$ presentations, by Prop. 2.3 it has the model amalgamation property. By the natural isomorphisms $i$ between the categories of models of the diagrams and the corresponding categories of model homomorphisms we have that the model amalgamation property for the outer square $\left[\iota_{\varphi_{1}}\left(h_{1}\right), \iota_{\varphi_{2}}\left(h_{2}\right), \iota_{\theta_{1}}\left(g_{1}\right), \iota_{\theta_{2}}\left(g_{2}\right)\right]$ is equivalent to the model amalgamation property the for considered pushout square of $\mathcal{I}^{2}$ signature morphisms. But the latter model amalgamation property holds by Prop. 3.2, hence the outer square $\left[\iota_{\varphi_{1}}\left(h_{1}\right), \iota_{\varphi_{2}}\left(h_{2}\right), \iota_{\theta_{1}}\left(g_{1}\right), \iota_{\theta_{2}}\left(g_{2}\right)\right]$ has the model amalgamation property too. Now by Prop. 2.2 applied for $\mathcal{I}^{\text {pres }}$, we obtain that the mediating presentation morphism $\gamma$ is conservative.

Since by the hypothesis $\left(\varphi_{1}, h_{1}\right) \in \mathcal{L}^{2}$ and $\left(\varphi_{2}, h_{2}\right) \in \mathcal{R}^{2}$ we have that $\iota_{\varphi_{1}}\left(h_{1}\right) \in \mathcal{L}$ and $\iota_{\varphi_{2}}\left(h_{2}\right) \in \mathcal{R}$. By Prop. 2.5 it follows that the inner square $\left[\iota_{\varphi_{1}}\left(h_{1}\right), \iota_{\varphi_{2}}\left(h_{2}\right), \phi_{1}, \phi_{2}\right]$ is a CRI square and by Prop. 2.4 it further follows that the outer square $\left[\iota_{\varphi_{1}}\left(h_{1}\right), \iota_{\varphi_{2}}\left(h_{2}\right), \iota_{\theta_{1}}\left(g_{1}\right), \iota_{\theta_{2}}\left(g_{2}\right)\right]$ is a CRI square too. By Fact 3.1 we deduce that the pushout square of $\mathcal{I}^{\prime}$-signature morphisms $\left[\left(\varphi_{1}, h_{1}\right),\left(\varphi_{2}, h_{2}\right),\left(\theta_{1}, g_{1}\right),\left(\theta_{2}, g_{2}\right)\right]$ is a CRI square, which completes this proof.

Corollary 4.1. $F O L^{2}$ has both Craig-Robinson $\left\langle\mathcal{S}, \mathbb{S}_{i} F O L^{2}\right\rangle$ - and $\left\langle\mathbb{S} i g^{F O L^{2}}, \mathcal{S}\right\rangle$-interpolation for $\mathcal{S}$ the class of $F O L^{2}$-signature morphisms $(\varphi, h)$ with $\varphi$ injective on the sort symbols.

Proof. For the standard system of diagrams for $F O L$ (see Ex. 2.3) it is easy to note that if $\varphi$ is injective on the sort symbols then $\iota_{\varphi}(h)$ is also injective on the sort symbols. The conclusion of the corrolary follows by applying Thm. 4.1 for the FOL interpolation properties presented in Ex. 2.4.

Corollary 4.2. $H C L^{2}$ has Craig-Robinson $\left\langle\mathcal{L}, \operatorname{Sig}^{H C L^{2}}\right\rangle$-interpolation for $\mathcal{L}$ the class of $H C L^{2}$ signature morphisms $(\varphi, h)$ with $\varphi$ a $(i e *) H C L$-signature morphism and $h$ a surjective model homomorphism.

Proof. We first show that the hypotheses on $(\varphi, h)$ imply that $\iota_{\varphi}(h)$ is an $(i e *) H C L$-signature morphism, where $\iota$ is the standard system of diagrams from $H C L$ presented in Ex. 2.3. Let $\varphi:(S, F, P) \rightarrow\left(S^{\prime}, F^{\prime}, P^{\prime}\right)$ and $h:\left.M \rightarrow M^{\prime}\right|_{\varphi}$. Because $\varphi^{\text {st }}$ is injective we obtain immediately that $\iota_{\varphi}(h)^{\text {st }}$ is injective too. The encapsulation property for $\iota_{\varphi}(h)^{\mathrm{op}}$, which we have to show here, means that for any $\sigma^{\prime} \in\left(F_{M^{\prime}}^{\prime}\right)_{w^{\prime} \rightarrow s^{\prime}}$ with $s^{\prime}=\varphi(s)$ (by the injectivity of $\varphi^{\text {st }}$ we 
have that $s$ is unique) there exists $\sigma \in\left(F_{M}\right)_{s}$ such that $\sigma^{\prime}=\iota_{\varphi}(h)^{\mathrm{op}}(\sigma)$. We distinguish two cases:

1 When $\sigma^{\prime} \in F_{w^{\prime} \rightarrow s^{\prime}}^{\prime}$ the property follows by the encapsulation property for $\varphi^{\mathrm{op}}$.

2 When $\sigma^{\prime} \in M_{s^{\prime}}^{\prime}$, because $M_{s^{\prime}}^{\prime}=\left(M^{\prime} \uparrow_{\varphi}\right)_{s}$ and $h_{s}$ is surjective there exists $\sigma \in M_{s}$ such that $h_{s}(\sigma)=\sigma^{\prime}$. Then $\iota_{\varphi}(h)^{\mathrm{op}}(\sigma)=\sigma^{\prime}$.

The conclusion of the corollary now follows by applying Thm. 4.1 for the $H C L$ interpolation properties presented in Ex. 2.5.

It may be useful to look now, from an applicational perspective, into the meaning of the conditions underlying the concrete interpolation results of Corollaries 4.1 and 4.2. Let us consider the rather important application of interpolation to the completeness of proof systems for structured specifications of (Borzyszkowski 2000). We consider this result in its reformed more general version presented in (Diaconescu 2008), which unlike the original version of (Borzyszkowski 2000), can be applied to $H C L$ as well, not only to $F O L$. Since Cor. 4.2 introduces stronger conditions than Cor. 4.1 let us focus on the $H C L^{2}$ example.

As readers may recall from (Borzyszkowski 2000; Diaconescu 2008), the above mentioned completeness result for structured specifications refers to specifications structured by three operators: sums, translations (i.e. renamings, not necessarily injective or surjective), and derivations (i.e. information hiding). Although the most common modularization operators in specification languages, such as imports, module sums, parameterized modules and their instantiations, can be explained only in terms of sums and translations, information hiding is quite important especially when related to the behavioural paradigm. The completeness theorem for structured specifications uses two special classes of signature morphisms, one for translations, and the other for the derivations. These classes of signature morphisms appear as parameters for the completeteness theorem. Within the context of Cor. 4.2, the translations are without restrictions, while the signature morphisms $(\varphi, h)$ used for the derivations are such that $\varphi$ is $(i e *)$ and $h$ is surjective. Note that the syntactic encapsulation condition given by $(i e *)$ and the 'no junk' semantic condition given by the surjectivity of $h$ fit well with the intuitions related to information hiding.

\section{Conclusions}

In this paper we have updated the semantics of predefined types at a general institution-independent level, we have proved a model amalgamation property for the resulting institution(s), we have obtained the concept of interpolation for predefined types as an instance of the general institutionindependent concept of interpolation to the institution(s) of predefined types, and we have formulated and, based on the above mentioned model amalgamation result, proved a general theorem lifting interpolation from arbitrary base institutions to the corresponding institutions of predefined types. We have illustrated the generic interpolation result with applications to predefined types over many-sorted first order logic and over Horn clause logic with equality. Similar applications may be developed for a multitude of other concrete situations in dependence on interpolation properties of the base logic. 


\section{References}

Eyal Amir and Sheila McIlraith. Improving the efficiency of reasoning through structure-based reformulation. In B.Y. Choueiry and T.Walsh, editors, Proceedings of the Symposium on Abstraction, Reformulation and Approximation (SARA'2000), volume 1864 of Lecture Notes in Artificial Intelligence, pages 247-259. Springer-Verlag Berlin Heidelberg, 2000.

Jan Bergstra, Jan Heering, and Paul Klint. Module algebra. Journal of the Association for Computing Machinery, 37(2):335-372, 1990.

Juan Bicarregui, Theodosis Dimitrakos, Dov Gabbay, and Tom Maibaum. Interpolation in practical formal development. Logic Journal of the IGPL, 9(2):231-243, 2001.

Tomasz Borzyszkowski. Generalized interpolation in CASL. Information Processing Letters, 76:19-24, 2001.

Tomasz Borzyszkowski. Logical systems for structured specifications. Theoretical Computer Science, 286(2):197-245, 2002.

Tomasz Borzyszkowski. Generalized interpolation in first-order logic. Fundamenta Informatica, 66(3):199-219, 2005.

Rod Burstall and Joseph Goguen. The semantics of Clear, a specification language. In Dines Bjorner, editor, 1979 Copenhagen Winter School on Abstract Software Specification, volume 86 of Lecture Notes in Computer Science, pages 292-332. Springer, 1980.

Chen-Chung Chang and H. Jerome Keisler. Model Theory. North Holland, Amsterdam, 1990.

Răzvan Diaconescu. Category-based semantics for equational and constraint logic programming, 1994. DPhil thesis, University of Oxford.

Răzvan Diaconescu. Category-based constraint logic. Mathematical Structures in Computer Science, 10(3):373-407, 2000.

Răzvan Diaconescu. Elementary diagrams in institutions. Journal of Logic and Computation, 14(5):651674,2004

Răzvan Diaconescu. Herbrand theorems in arbitrary institutions. Information Processing Letters, 90:29-37, 2004.

Răzvan Diaconescu. An institution-independent proof of Craig Interpolation Theorem. Studia Logica, 77(1):59-79, 2004.

Răzvan Diaconescu. Interpolation in Grothendieck institutions. Theoretical Computer Science, 311:439461, 2004

Răzvan Diaconescu. Institution-independent Model Theory. Birkhäuser, 2008.

Răzvan Diaconescu, Joseph Goguen, and Petros Stefaneas. Logical support for modularisation. In Gerard Huet and Gordon Plotkin, editors, Logical Environments, pages 83-130. Cambridge, 1993. Proceedings of a Workshop held in Edinburgh, Scotland, May 1991.

Theodosis Dimitrakos. Formal Support for Specification Design and Implementation. PhD thesis, Imperial College, 1998.

Theodosis Dimitrakos and Tom Maibaum. On a generalized modularization theorem. Information Processing Letters, 74:65-71, 2000.

Joseph Goguen and Rod Burstall. Institutions: Abstract model theory for specification and programming. Journal of the Association for Computing Machinery, 39(1):95-146, 1992.

Joseph Goguen and José Meseguer. Models and equality for logical programming. In Hartmut Ehrig, Giorgio Levi, Robert Kowalski, and Ugo Montanari, editors, Proceedings, TAPSOFT 1987, volume 250 of Lecture Notes in Computer Science, pages 1-22. Springer, 1987.

Joseph Goguen, James Thatcher, Eric Wagner, and Jesse Wright. Initial algebra semantics and continuous algebras. Journal of the Association for Computing Machinery, 24(1):68-95, January 1977. An early version is "Initial Algebra Semantics", with James Thatcher, IBM T.J. Watson Research Center, Report RC 4865, May 1974. 
Daniel Găină and Andrei Popescu. An institution-independent proof of Robinson consistency theorem. Studia Logica, 85(1):41-73, 2007.

Ranjit Jhala, Rupak Majumdar, and Ru-Gang Xu. State of the union: Type inference via Craig interpolation. In Tools and Algorithms for the Construction and Analysis of Systems, volume 4424 of Lecture Notes in Computer Science, pages 553-567. Springer, 2007.

Oliver Kutz and Till Mossakowski. Modules in transition. Conservativity, Composition, and Colimits. In Proceedings, Second International Workshop on Modular Ontologies, 2007.

Saunders Mac Lane. Categories for the Working Mathematician. Springer, second edition, 1998.

S. Maehara. On the interpolation theorem of Craig. Sugaku, pages 235-237, 1961-1962. In Japanese.

Sheila McIlraith and Eyal Amir. Theorem proving with structured theories. In Proceedings of the 17th Intl. Conf. on Artificial Intelligence (IJCAI-01), pages 624-631, 2001.

Kenneth McMillan. Applications of Craig interpolants in model checking. In Proceedings TACAS'2005, volume 3440 of Lecture Notes in Computer Science, pages 1-12. Springer, 2005.

José Meseguer. General logics. In H.-D. Ebbinghaus et al., editors, Proceedings, Logic Colloquium, 1987, pages 275-329. North-Holland, 1989.

Greg Nelson and Derek Oppen. Simplication by cooperating decision procedures. ACM Trans. Program. Lang. Syst., 1(2):245-257, 1979.

Derek Oppen. Complexity, convexity and combinations of theories. Theoretical Computer Science, 12:291302,1980

Marius Petria and Răzvan Diaconescu. Abstract Beth definability in institutions. Journal of Symbolic Logic, 71(3):1002-1028, 2006.

Pieter-Hendrik Rodenburg. A simple algebraic proof of the equational interpolation theorem. Algebra Universalis, 28:48-51, 1991.

Donald Sannella and Andrzej Tarlecki. Specifications in an arbitrary institution. Information and Control, 76:165-210, 1988.

Joseph Shoenfield. Mathematical Logic. Addison-Wesley, 1967.

Andrzej Tarlecki. Bits and pieces of the theory of institution. In David Pitt, Samson Abramsky, Axel Poigné, and David Rydeheard, editors, Proceedings, Summer Workshop on Category Theory and Computer Programming, volume 240 of Lecture Notes in Computer Science, pages 334-360. Springer, 1986.

Andrzej Tarlecki. On the existence of free models in abstract algebraic institutions. Theoretical Computer Science, 37:269-304, 1986.

Andrzej Tarlecki, Rod Burstall, and Joseph Goguen. Some fundamental algebraic tools for the semantics of computation, part 3: Indexed categories. Theoretical Computer Science, 91:239-264, 1991.

Paulo Veloso. On pushout consistency, modularity and interpolation for logical specifications. Information Processing Letters, 60(2):59-66, 1996. 